\title{
Verwaltung in der Öffentlichkeit: Zur Bedeutung kommunikativer Problemstellungen in den Zeiten technologisch induzierten Medienwandels
}

\section{Klaus Kocks, Susanne Knorre und Jan Niklas Kocks}

„Administration: An ingenious abstraction in politics, designed to receive the kicks and cuffs due to the premier or president. A man of straw, proof against bad-egging and dead-catting" Ambrose Bierce (1906).

Der Zwang zur beinahe permanenten öffentlichen Kommunikation ist kein originäres Phänomen der modernen Mediengesellschaft, auch wenn diese die Salienz des Phänomens auf ein neues Niveau befördert hat. Schon zu früheren Zeiten musste sich Herrschaft im öffentlichen Raum kommunikativ legitimieren und so finden sich Frühformen politischer und administrativer Öffentlichkeitsarbeit bereits zu Zeiten, in denen der Begriff als solcher noch nicht einmal geprägt war (Birkner 2018; Fröhlich 1996).

Mit zunehmender Medialisierung - der Durchdringung aller Lebensbereiche und insbesondere auch der Politik durch mediale Funktionslogiken (Imhof 2006; Strömbäck 2008) - und dem nachfolgenden und zugleich eng damit verbundenen Prozess der Digitalisierung und medialen Hybridisierung (Chadwick 2013) haben

K. Kocks $\cdot$ J. N. Kocks $(\bowtie)$

CATO Sozietät für Kommunikationsberatung $\mathrm{GmbH}$, Horbach und Berlin, Deutschland

E-Mail: j.n.kocks@fu-berlin.de

K. Kocks

E-Mail: ceterum.censeo@mailtrack.de

S. Knorre

Institut für Kommunikationsmanagement, Hochschule Osnabrück, Campus Lingen,

Lingen (Ems), Deutschland

E-Mail: s.knorre@fh-osnabrueck.de 
sich die kommunikativen Erwartungen an Akteure ${ }^{1}$ des politisch-administrativen Systems und zum Teil auch deren Möglichkeiten stetig erweitert. Dies gilt im staatlichen Bereich zweifelsohne für den zunächst zurückhaltend (Canél und Sanders 2012), in den letzten Jahren jedoch zunehmend wissenschaftlich untersuchten und empirisch vermessenen Bereich der Gubernative (u. a.: Borucki 2014; DePaula et al. 2017; Garland et al. 2018; Kocks 2016; Raupp und Kocks 2018; Sanders et al. 2011). Dies gilt allerdings zunehmend auch für den bis dato noch kaum untersuchten Bereich der Administrative.

Hatte Kurt Tucholsky seinerzeit noch ironisierend davon gesprochen, dass es das deutsche Schicksal sei, vor einem Schalter zu stehen und das deutsche Ideal hinter eben jenem Schalter zu sitzen (Tucholsky 1930), eine klare Ironisierung obrigkeitsstaatlichen Denkens und autoritärer Verwaltungsstrukturen im Deutschen Reich und der Weimarer Republik, so haben sich das Bild von und die Erwartungen an moderne Verwaltung grundlegend gewandelt, insbesondere auch in kommunikativer Hinsicht. Der demokratische Souverän erwartet von staatlichen Institutionen Responsivität und Transparenz (Fairbanks et al. 2007; Jaeger und Bertot 2010; Patzelt 2005), er möchte über ihr Handeln informiert werden und seine Anliegen im Idealfall niedrigschwellig und direkt an sie herantragen. Dies gilt, allen bürokratischen Routinen zum Trotz, auch für den Bereich der öffentlichen Verwaltung.

Auch interne Anspruchsgruppen der öffentlichen Verwaltungen haben andere kommunikative Ansprüche, als dies traditionell der Fall war. Mag die interne Organisationsstruktur dieser Institutionen auch weiterhin eine hierarchische sein, öffentliche Verwaltung kann und soll im Rechtsstaat mitnichten die Strukturen und Prozesse moderner Start-Ups emulieren, so bedeutet dies dennoch nicht mehr notwendigerweise eine rein auf schriftlichen Dienstanweisungen und Umlaufmappen basierende Form interner Kommunikation. Was sich im Bereich der Wirtschaft zunehmend etabliert und, gerade aus der Sicht effizienten kommunikationsbasierten Managements auch bewährt (Buchholz und Knorre 2019), hält langsam aber sicher auch Einzug in den Bereich der öffentlichen Verwaltung: professionelle, direkte und responsive interne Kommunikation mit den verschiedenen Anspruchsgruppen innerhalb des behördlichen Apparats.

Die technischen Voraussetzungen für neue Formen direkter und responsiver in- und externer Kommunikation der öffentlichen Verwaltung sind, dem technologisch induzierten Medienwandel und der Professionalisierung der politischen

\footnotetext{
${ }^{1}$ In diesem Sammelband wird aus Gründen der besseren Lesbarkeit das generische Maskulinum verwendet. Hiermit sind sowohl Frauen als auch Männer gemeint, wie auch alle Menschen, die sich keinem dieser Geschlechter zuordnen.
} 
Kommunikation sei Dank (Chadwick 2013; Kamps 2007; Lilleker und KocMichalska 2016), besser denn je. Professionelle (Fach-) Kommunikatoren verfügen im Zeitalter der Digitalisierung über eine Vielzahl von Kommunikationsmitteln und -kanälen, mit denen sie ihre diversen Zielgruppen spezifisch adressieren können.

Zur gleichen Zeit ergeben sich aber auch vielfältige neue Problemstellungen. Manche sind im rechtlichen Bereich zu verorten: Was kann und darf öffentliche Verwaltung kommunizieren? Wie kann Problemen bewusster Kommunikationssabotage, etwa durch sogenanntes Trolling, begegnet werden, ohne Bürger von der Kommunikation ihrer staatlichen Institutionen auszuschließen (Engeler 2017; siehe auch Wissenschaftliche Dienste Ausarbeitung WD3-3000-044/18 2018)? Welche Informationspflichten und Regeln des Datenschutzes gilt es zu beachten?

Andere Problemstellungen ergeben sich im Spannungsfeld zwischen behördlichen Organisationslogiken und den Funktionslogiken insbesondere sozialer Medien: Wie lassen sich behördliche Arbeitsabläufe und digitale Kommunikationsroutinen in Einklang bringen? Welche notwendigen Freiheiten brauchen behördliche Kommunikatoren und wie lassen sich diese gewährleisten? Auch im Bereich der Evaluation bestehen Desiderata: Nach welchen Standards kann behördliche Kommunikation evaluiert werden, insbesondere im Hinblick auf Fragen der Zielgruppenerreichung und -reichweite? Auch der Rückgriff auf externe Expertise ist zu problematisieren: Welche Formen der Kommunikationsberatung sind verfügbar und adäquat für diese spezifische Form öffentlicher Kommunikation? Inwieweit kann und darf öffentliche Verwaltung auf externe Berater setzen? Wie kann all das gelingen, ohne die Fehler eines ritualisierten und kostenintensiven Kommunikationscontrollings nun im öffentlichen Sektor zu wiederholen?

Diese und andere Fragestellungen entstammen mitnichten nur dem akademischen Elfenbeinturm, sie sind gegenwärtig vielmehr Gegenstand umfangreicher, zum Teil auch medial geführter Debatten. Da sucht die Berliner Polizei die Routinen bloßer Verlautbarungskommunikation zu durchbrechen und bedient sich zur Schilderung realer Einsätze narrativer Mittel, da sucht sie ihren Auftritt in einem vor allem bildgetriebenen Medium dadurch aufzulockern, dass sie ihre Rezipienten in die Suche nach dem abendlichen Flirt eines Kollegen einbindet. Beides mit Sicherheit medienadäquate Kommunikation - aber auch behördenadäquat? Die Hauptstadtpresse, sonst gerne auf Seiten derer, die antiquierte, langsame und wenig ansprechende Behördenkommunikation bemängeln, erging sich in umfangreicher Kritik (exemplarisch siehe Aydemir 2019; Geisler 2019), zum Teil gut begründet, zum Teil eher durch Häme getrieben. Der Einsatz externer Expertise, in den Public Relations seit langem etabliert und umfangreich bewährt, er steht in Zeiten, in denen das größte deutsche Nachrichtenmagazin mit dem Titel „Die 
fünfte Gewalt: Wie sich der deutsche Staat globalen Beraterfirmen ausliefert" aufmacht (Der Spiegel 2019) latent unter Verdacht: Werden hier notwendige bzw. nützliche Ressourcen und Kompetenzen von außerhalb zielgerichtet und zum Vorteil der öffentlichen Verwaltung eingesetzt, oder liefern sich demokratisch legitimierte Instanzen undurchsichtigen und profitorientierten Akteuren aus, die sich freihändig an öffentlichen Mitteln zu bedienen suchen? Zeitgemäße Behördenkommunikation wird unter den hier geschilderten Bedingungen immer häufiger zu einer heiklen Angelegenheit für die (Fach-) Kommunikatoren und die Führungsverantwortlichen in den Behördenleitungen, die sich in einem Spannungsfeld zwischen stetig zunehmenden kommunikativen Ansprüchen ihrer Rezipienten einerseits und institutionellen, normativen und rechtlichen Schranken andererseits wiederfinden.

Aber nicht nur aus berufspraktischer Perspektive verdient das Feld der Behördenkommunikation unter den Bedingungen des technologisch induzierten Medienwandels und der zunehmenden Digitalisierung sämtlicher Behördenbeziehungen Aufmerksamkeit. Im Bereich der (politischen) Kommunikationsforschung lassen sich hier noch immer sehr deutliche Desiderata identifizieren. Lag lange Zeit vor allem die Kommunikation von politischen Parteien und Nichtregierungsorganisationen im Fokus ihrer Aufmerksamkeit, so hat sie in den letzten Jahren auch den zuvor noch reichlich stiefmütterlich behandelten Bereich der Regierungskommunikation und staatlichen Öffentlichkeitsarbeit in den Blick genommen (Canél und Sanders 2012; Lee et al. 2011; Raupp und Kocks 2018). $\mathrm{Ob}$ und wie Kommunikatoren aus dem Kernbereich der Exekutive mittels moderner und multipler Kanäle mit dem Bürger als demokratischem Souverän kommunizieren ist für die politische Kommunikationsforschung zunehmend relevant geworden. Empirische Arbeiten widmen sich unter anderem der Digitalisierung der Öffentlichkeitsarbeit in diesem Bereich (u. a.: Chen et al. 2007; DePaula et al. 2017), zum Teil auch aus komparativer Perspektive (u. a.: Borucki 2018; Sanders et al. 2011; Vogel 2010). Andere Arbeiten nehmen die Beziehungen zwischen Regierungskommunikation und politischem Journalismus im Online-Zeitalter in den Blick (u. a.: Burgert 2009; Kocks und Raupp 2018; Rawnsley und Gong 2011). Für den Bereich der Administrative stehen demgegenüber noch immer verhältnismäßig wenige empirische Arbeiten zur Verfügung (Schatz 2008), neben diesen liegen einige zumeist ältere Arbeiten an der Schnittstelle von Forschung und Berufspraxis vor (u. a.: Antonoff 1971; Bischoff 1978; Peter 1992; Stockinger 1983; Wimmer 1982). Angesichts der Bedeutung öffentlicher Verwaltung für das demokratische System und des Umfangs ihrer Kommunikationsleistungen ist dieser Zustand zumindest unbefriedigend. 
Der vorliegende Band unternimmt daher den Versuch, den Themenkomplex , Verwaltung in der Öffentlichkeit' aus unterschiedlichen wissenschaftlichen und berufspraktischen Perspektiven zu diskutieren und empirisch zu untersuchen. Er möchte damit denjenigen, die täglich im Spannungsfeld der Innen- und Außenkommunikation der öffentlichen Verwaltung tätig sind, eine Orientierung an die Hand geben und zugleich einen Anstoß dafür bieten, das bis dato nur sehr zurückhaltend erforschte Feld aus der Perspektive von Kommunikations-, Politik- und Rechtswissenschaft weiter zu erschließen.

Im ersten Abschnitt des Bandes wird der Themenkreis zunächst aus verschiedenen theoretischen Perspektiven diskutiert: Eingangs widmet sich Peter Szyszka in seinem Beitrag der Frage, wie sich die Öffentlichkeitsarbeit der öffentlichen Verwaltung kommunikationswissenschaftlich verorten lässt und wo die besonderen Charakteristika und Herausforderungen dieser Form öffentlicher Kommunikation liegen. Dabei wird zunächst eine organisatorische Perspektive eingenommen, sodann werden die Spezifika dieser Form der Öffentlichkeitsarbeit herausgearbeitet. Im Weiteren diskutiert Szyszka die Frage, wie öffentliche Verwaltungskommunikation den Bedingungen des medialen und des damit verbundenen gesellschaftlichen und politischen Wandels Rechnung tragen kann. Der folgende Beitrag von Susanne Knorre fokussiert dann auf die organisationsinterne Kommunikation und deren Einordnung in das Managementparadigma, das die öffentliche Verwaltung jahrzehntelang dominiert hat. Knorre führt aus, wie es auch unter den Bedingungen öffentlicher Verwaltung gelingen kann, mit den Konzepten einer kommunikationszentrierten Führung und eines agilen Managements die internen Ressourcen von Behörden effektiver zu nutzen und damit die Effektivitäts- und Effizienzpotenziale von (großen) Verwaltungsorganisationen zu heben. Dabei steht insbesondere die Mobilisierung der Mitarbeitenden in Behörden im Fokus, die nicht zuletzt mit den Mitteln der internen Kommunikation geleistet werden muss.

Aus der Perspektive der Rechtswissenschaft arbeitet Till Dunckel in seinem Beitrag die spezifischen rechtlichen Problemstellungen heraus, die sich für öffentlich kommunizierende Verwaltungen ergeben. Dabei werden zunächst die wichtigsten Regelungen erörtert, nach denen öffentliche Verwaltung zur Kommunikation verpflichten sein kann. Anschließend wird diskutiert, unter welchen Voraussetzungen eine Behörde berechtigt ist, auch ohne Verpflichtung aktiv mit der Öffentlichkeit zu kommunizieren und welche spezifischen rechtlichen Grenzen für die Inhalte der Verwaltungskommunikation gelten. Der anschließende Beitrag von Reinhold Fuhrberg widmet sich dem Themengebiet der Verwaltungskommunikation aus verhaltensökonomischer Sicht, wobei der Schwerpunkt 
der Betrachtung auf dem Phänomen des sogenannten ,Nudgings‘ liegt, bei dem staatliche Stellen zur Verhaltens- und Entscheidungssteuerung kommunizieren. Der Beitrag systematisiert dessen verhaltensökonomische Grundlagen und stellt kommunikative Vorgehensweisen und Anwendungsbeispiele vor. Aufgrund der konträr geführten öffentlichen Diskussion in diesem Bereich erfolgt sodann eine normative Einordnung. Abschließend wird der Blick daraufgelegt, was das Kommunikationsmanagement hinsichtlich der Ausgestaltung strategischer Kommunikation aus den Erkenntnissen der Verhaltensökonomie lernen kann.

Im zweiten Abschnitt des Bandes werden ausgewählte empirische Befunde zur Verwaltungskommunikation vorgestellt und diskutiert: Jan Niklas Kocks und Juliana Raupp untersuchen in ihrem Beitrag empirisch das besondere Gebiet der überfraktionellen parlamentarischen Öffentlichkeitsarbeit, jene Form der politischen Kommunikation, in der nicht Abgeordnete oder Fraktionen sich an die Öffentlichkeit wenden, sondern das Parlament in seiner Eigenschaft als demokratische Institution. Wie gehen deutsche Parlamente mit den Herausforderungen der Digitalisierung um und welche Problemstellungen ergeben sich insbesondere im Bereich der Evaluation von Online-Öffentlichkeitsarbeit? Der Beitrag diskutiert die Befunde einer komparativen Inhaltsanalyse und nimmt spezifische evaluative Fragestellungen der Öffentlichkeitsarbeit öffentlicher Verwaltung in den Blick.

Im anschließenden Beitrag diskutiert Dimitrij Umansky die Befunde einer qualitativen Fallstudie zur Kommunikation kommunaler Verwaltungsbehörden hinsichtlich der Öffentlichkeitsbeteiligung: Welche Beteiligungs- und Wahrnehmungsziele verfolgen kommunale Verwaltungsbehörden in ihrer öffentlichen Kommunikation? Welche Konflikte ergeben sich zwischen diesen Zielstellungen und wie wirken sich diese auf die begleitende Öffentlichkeitsarbeit aus? Anhand einer Befragung von Kommunalvertretern und kommunalen Öffentlichkeitsarbeitern in Niedersachsen zum Übertragungsnetzausbau diskutiert Umansky diese Fragen und arbeitet auf dieser Basis die spezifischen Herausforderungen kommunaler Öffentlichkeitsarbeit im Rahmen von Beteiligungsverfahren heraus. Lars Rademacher, Heiko Kretschmer und Klaus Lintemeier nehmen in ihrem Beitrag den Problemkreis der Öffentlichkeitsbeteiligung bei Infrastrukturprojekten in den Blick. Wie integriert die Politik in Deutschland auf kommunaler, regionaler oder Länderebene partizipative Elemente in geplante Bau- und Infrastrukturprojekte, welche Erwartungen haben die Beteiligten hinsichtlich ihrer Umsetzung? Wo liegen, aus Sicht der Beteiligten, Chancen und Fallstricke einer kommunikativen Integration der Bürger in Vorbereitung und Durchführung? Der Beitrag diskutiert die Befunde einer empirischen Befragungsstudie und formuliert darauf aufbauend eine Reihe von Thesen zur Zukunft der Öffentlichkeitsbeteiligung in diesem Bereich. 
Im dritten Abschnitt des Bandes rücken schließlich die praxisbezogenen Perspektiven des Kommunikationsmanagements und der Kommunikationsberatung in den Blick: An der Schnittstelle von Wissenschaft und Praxis beschreibt Klaus Kocks in seinem Beitrag das Verhältnis von Verwaltung auf der einen Seite und Politik- und Kommunikationsberatung auf der anderen Seite. Wie und unter welchen Bedingungen kann öffentliche Verwaltung von externer Expertise profitieren? Wo liegen die spezifischen Fallstricke? Verwaltungshandeln ist, so Kocks, immer stärker auch kommunikatives Handeln und Amtsträger finden sich in ihrem Bemühen um (kommunikative) Vermittlung von staatlichen und bürgerlichen Interessen oftmals sprichwörtlich zwischen Baum und Borke wieder. Die möglichen Hilfestellungen durch externe Expertise und die im Gegenstand begründeten Grenzen dieser sind Thema seines Beitrages.

Im folgenden Beitrag diskutiert Andreas Möser die Herausforderungen und Chancen der Digitalisierung für die kommunale Öffentlichkeitsarbeit aus berufspraktischer Perspektive am Beispiel verschiedener Städte und Kommunen. Er konstatiert eine zunehmende Prägung der Praxis kommunaler Kommunikation durch rapide Veränderungen der Rahmenbedingungen gleichermaßen für Politik und Verwaltung, die klassischen Lokalmedien, Bürger und die zwischen allen agierenden Kommunikationsteams. Das Internet und die sozialen Netze haben, so Möser, die Kommunikationslage in diesem Bereich grundlegend verändert. Die besonderen Herausforderungen der sozialen Medien für die Öffentlichkeitsarbeit im Bereich der öffentlichen Verwaltung diskutiert schließlich Daniela Vey in ihrem Beitrag aus einer praxisorientierten Perspektive: Welche Entscheidungen gilt es auf dem Weg hin zu einer funktionalen und angemessenen Online- und Social-Media-Präsenz zu treffen? Welche Ressourcen müssen eingeplant werden und wo liegen besondere organisatorische und sprachliche Hürden? Anhand mehrerer Fallbeispiele beleuchtet der Beitrag den Planungsprozess digitaler Öffentlichkeitsarbeit öffentlicher Verwaltung von der Konzeption bis hin zur Ausführung.

Es ist der Breite und Diversität der Diskussion eines bis dato noch wenig erschlossenen Forschungsfeldes geschuldet, dass hier im letzten Abschnitt des Bandes kein umfassendes, die einzelnen Fäden in Gänze aufnehmendes Fazit gezogen werden kann. Auch wäre dies angesichts der Intention dieses Bandes, einen ersten Anstoß zu Diskussionen in Wissenschaft und Praxis zu bieten, ein vermessenes und dem eigenen Anspruch zuwiderlaufendes Unterfangen. An seiner Stelle steht hier daher eine Diskussion zukünftiger Chancen und Risiken der Verwaltungskommunikation unter den Bedingungen der Digitalisierung und des technologisch induzierten Medienwandels. Susanne Knorre und Jan Niklas Kocks nehmen dazu die im Band diskutierten Entwicklungen in den Blick und 
diskutieren auf dieser Grundlage einige Szenarien, wie professionelle, effiziente und angemessene Verwaltungskommunikation sich in den nächsten zehn Jahren entwickeln könnte und welche Fallstricke sich hier möglicherweise ergeben. Welche Best- und Worst-Case-Szenarien sind denkbar und wo müssen Politik, politisches Kommunikationsmanagement und unter Umständen auch die Wissenschaft ansetzen, um einen Beitrag zu einer notwendigen und normativ auch geforderten effizienten Weiterentwicklung und Fundierung der Verwaltungskommunikation zu leisten?

Das Feld der Verwaltungskommunikation ist bis dato nur zurückhaltend erforscht, es verdient aber nach Ansicht der hier vertretenen Autoren und der Herausgeber zukünftig noch mehr Aufmerksamkeit, sei es aus der Perspektive der (empirischen) Wissenschaft, oder aus der Perspektive der Berufspraxis. Öffentliche Verwaltung ist, gerade auch unter den Bedingungen der Digitalisierung und des technologisch induzierten Medienwandels, immer auch Verwaltung in der Öffentlichkeit. Es ist an der Zeit, deren Kommunikationsleistungen öffentlich zu reflektieren und zu diskutieren. Die Beteiligten hoffen, dass der vorliegende Band dazu einen ersten kleinen Anstoß liefern kann.

Die Herausgeber danken allen Autoren für die gute Zusammenarbeit. Besonderer Dank gebührt Karin Siepmann für die gute Betreuung des Projekts seitens des Verlags.

Berlin \& Hannover im Frühjahr 2019

Klaus Kocks

Susanne Knorre Jan Niklas Kocks

\section{Literatur}

Antonoff, R. (1971). Wie man seine Stadt verkauft. Kommunale Werbung u. Öffentlichkeitsarbeit. Düsseldorf: VDI.

Aydemir, F. (23. Januar 2019). Aufruf bei Instagram: Polizeilich gesucht - Junge, schöne Frau. taz - Die tageszeitung. http://www.taz.de/!5565326/. Zugegriffen: 25. Apr. 2019.

Bierce, A. (1906). The cynic's word book. New York: Doubleday, Page \& Company.

Birkner, T. (2018). Regierungskommunikation und staatliche Öffentlichkeitsarbeit aus kommunikationshistorischer Perspektive. In J. Raupp, J. N. Kocks, \& K. Murphy (Hrsg.), Regierungskommunikation und staatliche Öffentlichkeitsarbeit. Implikationen des technologisch induzierten Medienwandels (S. 73-92). Wiesbaden: Springer VS.

Bischoff, D. (1978). Öffentlichkeitsarbeit der Gemeindeverwaltungen. Die Rolle des kommunalen Presseamtes im lokalen Kommunikationsraum. Münster: Regensberg.

Borucki, I. (2014). Regieren mit Medien. Auswirkungen der Medialisierung auf die Regierungskommunikation der Bundesregierung von 1982-2010. Opladen: Budrich. 
Borucki, I. (2018). Europäische Regierungschefs auf Facebook: Dialogische Offerten und Interaktion in Netzwerken rund um Fanpages. In J. Raupp, J. N. Kocks, \& K. Murphy (Hrsg.), Regierungskommunikation und staatliche Öffentlichkeitsarbeit. Implikationen des technologisch induzierten Medienwandels (S. 127-143). Wiesbaden: Springer VS.

Buchholz, U., \& Knorre, S. (2019). Interne Kommunikation und Unternehmensführung. Theorie und Praxis eines kommunikationszentrierten Managements. Wiesbaden: Springer Gabler.

Burgert, D. (2009). Politisch-mediale Beziehungsgeflechte. Ein Vergleich politikfeldspezifischer Kommunikationskulturen in Deutschland und Frankreich (3. Aufl.). Münster: LIT.

Canél, M. J., \& Sanders, K. (2012). Government communication: An emerging field in political communication research. In H. A. Semetko \& M. Scammell (Hrsg.), The Sage handbook of political communication (S. 85-96). London: Sage.

Chadwick, A. (2013). The hybrid media system. Politics and power. Oxford: University Press.

Chen, P., Gibson, R., Lusoli, W., \& Ward, S. (2007). Australian government and online-communication. In S. Young (Hrsg.), Government communication in Australia (S. 161-180). Cambridge: University Press.

DePaula, N., Dincelli, E., \& Harrison, T. M. (2017). Toward a typology of government social media communication. Democratic goals, symbolic acts and self-presentation. Government Information Quarterly, 35(1), 98-108. https://doi.org/10.1016/ j.giq.2017.10.003.

Der Spiegel. (2019). Die fünfte Gewalt. Wie sich der deutsche Staat globalen Beraterfirmen ausliefert. Titel des Hefts 5, 2019. https://magazin.spiegel.de/SP/2019/5/?utm_source= spon\&utm_campaign=heftkastenprint. Zugegriffen: 25. Apr. 2019.

Engeler, M. (2017). Der staatliche Twitter-Auftritt. Rechtliche Hürden und mögliche Lösungen. MultiMedia und Recht, 20(10), 651-656.

Fairbanks, J., Plowman, K. D., \& Rawlins, B. L. (2007). Transparency in government communication. Journal of Public Affairs, 7(1), 23-37.

Fröhlich, R. (1996). Auf der Suche nach dem PR-Urknall: Über einige Missverständnisse und Defizite in der PR-Geschichtsschreibung. In P. Szyszka (Hrsg.), Auf der Suche nach einer Identität. Annäherungen an eine Geschichte deutscher Öffentlichkeitsarbeit (S. 62-67). Berlin: Vistas.

Garland, R., Tambini, D., \& Couldry, N. (2018). Has government been mediatized? A UK perspective. Media, Culture and Society, 40(4), 496-513.

Geisler, S. (11. Januar 2019). Seltsamer Facebook-Post. Was mich an der Social-Media-Strategie der Polizei ärgert. Berliner Morgenpost. https://www.morgenpost.de/meinung/article216186857/Was-mich-an-der-Social-Media-Strategie-der-Polizei-aergert. html. Zugegriffen: 25. Apr. 2019.

Imhof, K. (2006). Mediengesellschaft und Medialisierung. Medien und Kommunikationswissenschaft, 54(2), 191-215.

Jaeger, P. T., \& Bertot, J. C. (2010). Transparency and technological change. Ensuring equal and sustained public access to government information. Government Information Quarterly, 27(4), 371-376.

Kamps, K. (2007). Politisches Kommunikationsmanagement. Grundlagen und Professionalisierung moderner Politikvermittlung. Wiesbaden: VS Verlag.

Kocks, J. N. (2016). Political media relations online as an elite phenomenon. Wiesbaden: Springer VS. 
Kocks, J. N., \& Raupp, J. (2018). Die Erklärungskraft sozialen Kapitals: Eine Analyse der Struktur politisch-medialer Kommunikationsnetzwerke unter Online-Bedingungen. In C. Eilders, O. Jandura, H. Bause, \& D. Frieß (Hrsg.), Vernetzung. Stabilität und Wandel gesellschaftlicher Kommunikation. Köln: Halem.

Lee, M., Neeley, G., \& Stewart, K. B. (Hrsg.). (2011). The practice of government public relations. Hoboken: CRC Press.

Lilleker, D. G., \& Koc-Michalska, K. (2016). What drives political participation? Motivations and mobilization in a digital age. Political Communication, 34(1), 1-23. https://doi. org/10.1080/10584609.2016.1225235.

Patzelt, W. J. (2005). Warum verachten die Deutschen ihr Parlament und lieben ihr Verfassungsgericht? Ergebnisse einer vergleichenden demoskopischen Studie. Zeitschrift für Parlamentsfragen, 36(3), 517-538.

Peter, J. (1992). Presse- und Öffentlichkeitsarbeit in der Kommune. Das Praktiker-Handbuch. München: Jehle.

Raupp, J., \& Kocks, J. N. (2018). Regierungskommunikation und staatliche Öffentlichkeitsarbeit aus kommunikationswissenschaftlicher Perspektive. In J. Raupp, J. N. Kocks, \& K. Murphy (Hrsg.), Regierungskommunikation und staatliche Öffentlichkeitsarbeit. Implikationen des technologisch induzierten Medienwandels (S. 7-23). Wiesbaden: Springer VS.

Rawnsley, G., \& Gong, Q. (2011). Political communications in democratic Taiwan. The relationship between politicians and journalists. Political Communication, 28(3), 323340. https://doi.org/10.1080/10584609.2011.572462.

Sanders, K., Canél, M. J., \& Holtz-Bacha, C. (2011). Communicating governments. A three country comparison of how governments communicate with citizens. International Journal of Press and Politics, 16(4), 523-547.

Schatz, H. (2008). Regieren in der Mediengesellschaft: Zur Medialisierung von Politik und Verwaltung in der Bundesrepublik Deutschland. In W. Jann \& K. König (Hrsg.), Regieren zu Beginn des 21. Jahrhunderts (S. 127-174). Tübingen: Mohr Siebeck.

Stockinger, H. G. (1983). Bürgernahe kommunale Öffentlichkeitsarbeit. Hilfen für Mandatsträger und Verwaltung. München: Hanns-Seidel-Stiftung.

Strömbäck, J. (2008). Four phases of mediatization. An analysis of the mediatization of politics. The International Journal of Press/Politics, 13(3), 228-246.

Tucholsky, K. (1930). Schnipsel. Die Weltbühne. Berlin: Verlag der Weltbühne.

Vogel, M. (2010). Regierungskommunikation im 21. Jahrhundert. Ein Vergleich zwischen Großbritannien, Deutschland und der Schweiz. Baden-Baden: Nomos.

Wimmer, R. (1982). Kommunale Alternativen zur Lokalpresse. Rechtliche Aspekte neuer gemeindlicher Öffentlichkeitsarbeit. Neue Juristische Wochenschrift, 2793-2800.

Wissenschaftliche Dienste Ausarbeitung WD3-3000-044/18. (2018). Zugang zur Öffentlichkeitsarbeit der Polizei in sozialen Medien („Twitter“). https://www.bundestag.de/resource/blob/547012/39657aa84201156a8cb7e1e12be7cd14/WD-3-044-18-pdfdata.pdf. Zugegriffen: 25. Apr. 2019. 
Open Access Dieses Kapitel wird unter der Creative Commons Namensnennung 4.0 International Lizenz (http://creativecommons.org/licenses/by/4.0/deed.de) veröffentlicht, welche die Nutzung, Vervielfältigung, Bearbeitung, Verbreitung und Wiedergabe in jeglichem Medium und Format erlaubt, sofern Sie den/die ursprünglichen Autor(en) und die Quelle ordnungsgemäß nennen, einen Link zur Creative Commons Lizenz beifügen und angeben, ob Änderungen vorgenommen wurden.

Die in diesem Kapitel enthaltenen Bilder und sonstiges Drittmaterial unterliegen ebenfalls der genannten Creative Commons Lizenz, sofern sich aus der Abbildungslegende nichts anderes ergibt. Sofern das betreffende Material nicht unter der genannten Creative Commons Lizenz steht und die betreffende Handlung nicht nach gesetzlichen Vorschriften erlaubt ist, ist für die oben aufgeführten Weiterverwendungen des Materials die Einwilligung des jeweiligen Rechteinhabers einzuholen.

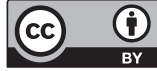

